\title{
OVEREXPRESSION OF INTERFERON PATHWAYS IN THE MUSCLE BIOPSIES OF THE ADULT PATIENTS WITH STABLE DERMATOMYOSITIS
}

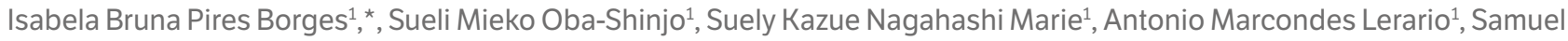
Katsuyuki Shinjo ${ }^{1}$

1. Faculdade de Medicina FMUSP, Universidade de São Paulo, São Paulo (SP), Brazil.

*Corresponding author: isapires@usp.br

\section{BACKGROUND}

Overexpression of interferon pathways in muscle and skin tissue, as well as in peripheral blood leukocytes from patients with dermatomyositis is correlated with the disease activity. However, studies focusing on the behavior of this pathway in muscle tissue of patients with stable dermatomyositis is still scarce, which motivated this study.

\section{METHODS}

This is a cross-sectional study in which 15 patients with defined dermatomyositis (EULAR/ACR 2017) and disease stable (according to the IMACS set scores) were evaluated. Patients were age, gender, and body mass matched with 10 health individuals (control group). Vastus lateralis muscle biopsies were performed to collect muscle tissues in order to analyze the whole transcriptome (RNA-Seq). The results were analyzed considering expression alterations (hyper or hypo-expressed), using fold change $\geq 0.4$ and $\leq-0.4$ ( $\log$ in base 2 ) and $p \leq 0.05$. Differentially expressed genes were analyzed with Web Gestalt (WEB-Based Gene SeT Analysis Toolkit using Over-Representation Analysis and the Gene Ontology functional database). Protein-protein interaction network analysis was performed using STRING.

\section{RESULTS}

Demographic parameters were comparable between the two groups. Four out of 15 patients were under prednisone therapy (three were using $5 \mathrm{mg} /$ day and one was using $15 \mathrm{mg} /$ day). All patients were treated with some immunosuppressive drug (azathioprine 2-3 mg/kg/day, methotrexate $15-25 \mathrm{mg} /$ week, mycophenolate mofetil $2-3 \mathrm{~g} /$ day or leflunomide $20 \mathrm{mg} /$ day). The differential expression analysis of dermatomyositis and control muscle tissues resulted in 183 known genes, 92 downregulated and 91 upregulated. The enrichment of pathways resulted in 25 genes belonging to the interferon pathway, with four hypoexpressed genes and 21 overexpressed in dermatomyositis compared to the control group. Among them, we can highlight the overexpressed genes B2M, STAT1, GBP1, MX1, RSAD2, IFIT3, ISG15, PSMB8, HLA-A, and DDX58. These genes are upregulated by type I IFN, type II IFN, and are involved in the processing of class I MHC antigens.

\section{CONCLUSION}

These findings show that, even in this group of stable patients with low or no dose of glucocorticoids, there is an increase in the expression of genes related to the interferon pathway, suggesting the presence of a muscle inflammatory process. This information is very important for a better understanding of the disease evolution and follow up, and also the study of therapeutic targets in this group of patients.

\section{FUNDING}

FAPESP Grant No. 2019/11367-9 to IBPB, Grant No. 2019/11776-6 to SKS; CNPq Grant No. 303379/2018-9 to SKS.

\section{KEYWORDS}

Dermatomyositis, Interferon pathways, Transcriptome. 